\title{
OPEN Exploring synthetic biology for the development of a sensor cell line for automated bioprocess control
}

\begin{abstract}
Nikolas Zeh ${ }^{1,2 \bowtie}$, Melina Bräuer ${ }^{1,2}{ }^{2}$ Nadja Raab $^{1}$, René Handrick ${ }^{1}$ \& Kerstin Otte ${ }^{1}$
Unfavorable process conditions lead to adverse cultivation states, limited cell growth and thus hamper biotherapeutic protein production. Oxygen deficiency or hyperosmolality are among the most critical process conditions and therefore require continuous monitoring. We established a novel sensor $\mathrm{CHO}$ cell line with the ability to automatically sense and report unwanted process conditions by the expression of destabilized fluorescent proteins. To this end, an inducible real-time system to detect hypoxia by hypoxia response elements (HREs) of vascular endothelial growth factor (VEGF) origin reporting limitations by the expression of destabilized green fluorescent protein (GFP) was created. Additionally, we established a technique for observing hyperosmolality by exploiting osmotic response elements (OREs) for the expression of unstable blue fluorescent protein (BFP, FKBP-BFP), enabling the simultaneous automated supervision of two bioprocess parameters by using a dual sensor $\mathrm{CHO}$ cell line transfected with a multiplexable monitoring system. We finally also provided a fully automated in-line fluorescence microscopy-based setup to observe $\mathrm{CHO}$ cells and their response to varying culture conditions. In summary, we created the first $\mathrm{CHO}$ cell line, reporting unfavorable process parameters to the operator, and provided a novel and promising sensor technology accelerating the implementation of the process analytical technology (PAT) initiative by innovative solutions.
\end{abstract}

Optimized bioprocess conditions are essential to ensure strong cell growth, high productivity and good product quality during industrial biomanufacturing ${ }^{1}$. In particular, oxygen deficiency and hyperosmolality can be challenging parameters leading to unfavorable cellular behavior and significantly deteriorated performance ${ }^{2,3}$. In this context, a sufficient oxygen supply of at least 10 to $15 \% \mathrm{pO}_{2}{ }^{4}$ is crucial to maintain critical energy metabolism contributing to physical cellular functions such as cell growth or autophagy ${ }^{5-7}$. Additionally, osmolality of more than $0.42 \mathrm{osm} / \mathrm{kg}^{8}$ can negatively influence a bioprocess, as hyperosmolality is reported to reduce growth and cellular viability and impair product quality, as increased salt concentrations may promote aggregation or change the glycosylation pattern of a biotherapeutic ${ }^{9-11}$. To avoid these drawbacks, continuous monitoring of critical process parameters is required by $\mathrm{pO}_{2}$ probes for oxygen and manual offline measurements for osmolality to detect and adjust deviations and sustain a successful bioprocess.. In the case of offline measurements requiring cell culture samples, the risk of contamination is also increased in combination with the need for additional hands-on time for periodical data points. Moreover, as adverse culture conditions are not autonomously communicated be the cell line, the targeted values for $\mathrm{pO}_{2}$ or osmolality are defined by the operator demanding excessive testing for each generated cell line to explore optimal conditions ${ }^{12}$. Therefore, the aim of the current study was to generate a novel sensor cell line that autonomously senses and reports unfavorable cultivation conditions during bioprocessing at the molecular signal level to the operator, with the scope to simplify measurements and improves insights into the modulation of intracellular physiological stages by in-line monitoring in real time. Especially for modelling approaches a cell line reporting its current state may be an important improvement ${ }^{13}$.

In nature, mammalian cells exploit the use of response elements to sense and react to hostile environmental conditions ${ }^{14-16}$. Oxygen deficiency, for example, leads to the stabilization of hypoxia-inducing factor $1 \alpha(\mathrm{HIF}-1 \alpha)$, which dimerizes with its cofactor HIF- $1 \beta$ to interact with hypoxia response elements (HREs) and activates the expression of protective genes such as erythropoietin (EPO) or vascular endothelial growth factor A (VEGF-A) (Fig. 1B) to perpetuate cellular physiological functions ${ }^{17-20}$. 
A

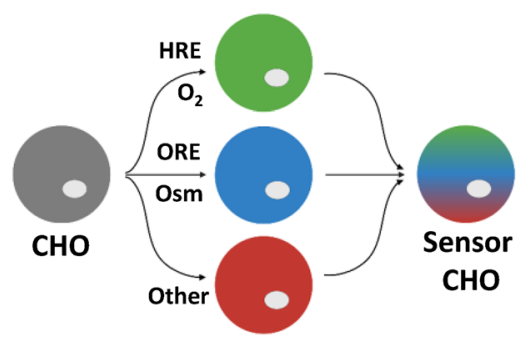

B
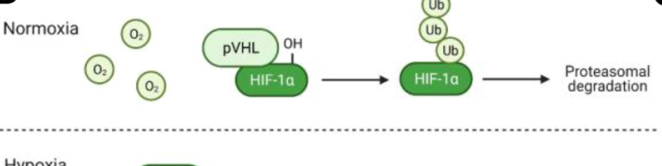

Hypoxia

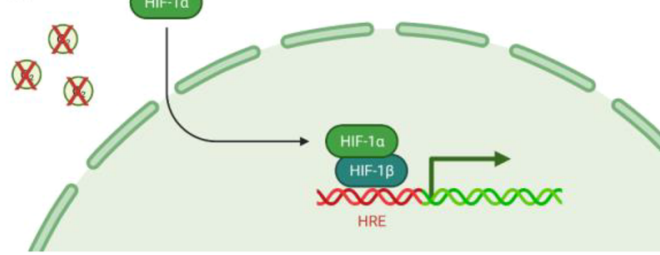

E

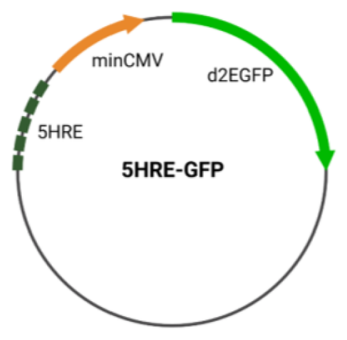

D

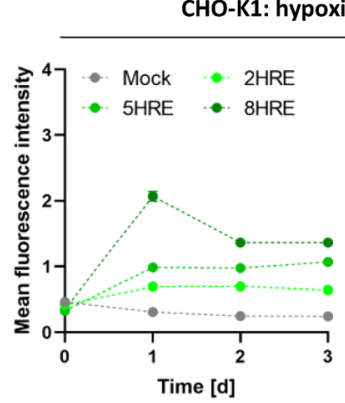

CHO-K1: hypoxia-induced fluorescence

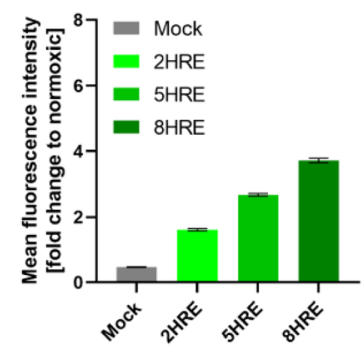

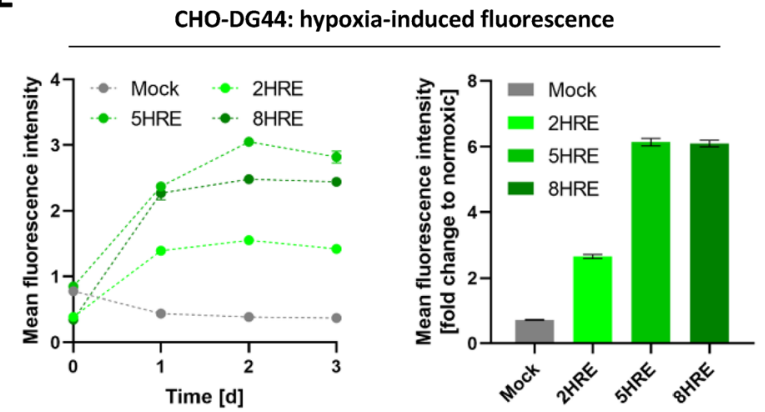

Figure 1. Establishment of a hypoxia-sensitive monitoring system. (A) Schematic overview of a CHO cell modified with different fluorescent expression vectors under the control of various response elements. (B) Hypoxia signaling pathway. (C) Schematic illustration of the hypoxia inducible d2GFP expression vector. (D) Flow cytometric analysis of shaken cultured CHO-K1 cells (left) and relative fold-change of mean fluorescence against the shaken cultured control (right). (E) Flow cytometric analysis of shaken cultured CHO-DG44 cells (left) and relative fold-change of mean fluorescence against the shaken cultured control (right).

A comparable mechanism preventing cells from receiving damage by external factors represents the response to hyperosmolality ${ }^{21,22}$. High salt concentrations lead to enhanced mRNA stabilization of the tonicity response element binding protein (TonEBP), better known as nuclear factor of activated T-cells 5 (NFAT5) ${ }^{23}$. As a consequence, enriched amounts of NFAT5 protein are phosphorylated and translocate into the nucleus for binding to osmotic response elements (OREs) of osmoprotective genes such as aldose reductase (AR) or secretin (SCT) and secretin receptor (SCTR $)^{22-25}$. In this context, NFAT5 features comparable competencies to HIF-1 $\alpha$, protecting cells from external influences and maintaining cellular functions by interacting with short, repetitive DNA sequences to coordinate target gene transcription.

In the current study, we aimed to generate a novel sensor cell line, reporting unfavorable cultivation conditions during bioprocessing by exploiting response elements for hypoxia and hyperosmolality inspired by nature. Specifically, we established an in-line oxygen reporter system in CHO cells by the expression of destabilized GFP (d2GFP) under the control of HREs of VEGF origin. Additionally, we showed the functionality of OREs of AR origin by the expression of unstable BFP (FKBP-BFP) to enable simultaneous detection of multiple process parameters. Finally, we were able to demonstrate independent functionality of the introduced monitoring systems and to set up an in-line automatically functional fluorescence microscope-based method to enable observation of $\mathrm{CHO}$ cells close to real time.

\section{Results}

Development of a genetic sensing system for oxygen deprivation. To simplify the measurement of unfavorable bioprocess conditions, we aimed to enable the $\mathrm{CHO}$ cells themselves to directly sense and report a changing environment within the cell culture vessel. Using a synthetic biology approach, we envisaged genetic response elements (REs) to drive destabilized fluorescent proteins. Different cellular fluorescence would then directly indicate changing bioprocess conditions (Fig. 1A). Since the available oxygen concentration is one of the main bioprocess conditions constantly monitored, we turned to the genetic control of oxygen sensing in mammalian cells. In this study, normoxia is defined as oxygen concentrations of $40 \%$ to $60 \% \mathrm{pO}_{2}$, while hypoxia describes $\mathrm{pO}_{2}$ values of $<15 \%$. The hypoxia response pathway is mainly controlled by hypoxia-inducing factor-1a (HIF-1a), which is degraded during normoxia. Under oxygen deprivation conditions, HIF-1 $\alpha$ is stabilized and binds together with HIF- $1 \beta$ to hypoxia response elements (HREs) to activate the expression of numerous genes (Fig. 1B). Previously, we showed the expression of relevant hypoxia response transcription factors in $\mathrm{CHO}$ cells ${ }^{4}$.

To establish a synthetic genetic sensing system for oxygen limitation in mammalian production cells, vectors were constructed linking a minimal CMV promoter to ensure expression of the fluorescent protein only under specified conditions to HREs driving the expression of destabilized GFP (d2GFP) (Fig. 1C). After stable transfection of expression vectors carrying two, five or eight canonical repetitive HREs into CHO-K1 and CHO-DG44 
cells, the functionality of the synthetic oxygen sensing system was evaluated by culturing cells under shaken or oxygen-limiting static conditions. Engineered CHO-K1 cells showed an increased fluorescence signal after one day of static cultivation, while mock cells lacking HREs in front of the minimal CMV maintained GFP fluorescence at a low basal level of 0.3 mean fluorescence (Fig. 1D). Furthermore, a correlation of GFP expression and the number of HRE repeats was observed, with the strongest signal for 8 HREs showing 2.1 mean fluorescence (Fig. 1D). When comparing statically (hypoxic) cultured CHO-K1 cells with their shaken (normoxic) cultured replica, an equal d2GFP signal was detected for CHO-K1-mock cells, but amplified expression of d2GFP (2- to 4-fold) was observed for the HRE construct harboring CHO cells (Fig. 1D). Similar observations were obtained for CHO-DG44 cells transfected with a mock- or HRE-encoding vector. While the mock cell line showed no inducible d2GFP expression under oxygen-limiting conditions ( 0.5 mean fluorescence), HREs led to a strong increase in the mean fluorescence signal of 1.6 to 3.1 (Fig. 1E). Compared to the shaken cultivated mock cell line, CHO-DG44 cells exhibited an even stronger up to 6-fold increase in d2GFP expression and outperformed $\mathrm{CHO}-\mathrm{K} 1$ cell lines (up to 4 -fold) grown under hypoxic conditions (Fig. 1D,E). In contrast to CHO-K1 cells showing the best inducibility of d2GFP expression with 8 HREs, the construct with 5 HREs led to the best results in CHO-DG44 cells (Fig. 1D,E). Additionally, basal expression of d2GFP under the leakage of minimal CMV was very low, with less than 0.2 mean fluorescence.

Expanding the sensing system to hyperosmolality. After developing a genetic sensor system for unfavorable bioprocess conditions, as exemplified by oxygen limiting conditions, we aimed to establish a synthetic multiplex sensor system. For bioprocess monitoring, osmolality is an important parameter; therefore, we searched for a genetic system for sensing and reporting. In this study, isotonic conditions are defined between 0.30 to $0.33 \mathrm{osm} / \mathrm{kg}$ and hyperosmolality is characterized by $>0.40 \mathrm{osm} / \mathrm{kg}$. In mammalian cells, osmolality is mainly sensed by phosphorylation states and expression levels of nuclear factor of activated T-cells 5 (NFAT5) ${ }^{24,25}$. During hyperosmolality, NFAT5 mRNA is stabilized, leading to higher amounts of NFAT5 protein, which enters the nucleus after phosphorylation as a transcription factor and activates gene expression by binding to osmolality response elements (OREs) (Fig. 2A) ${ }^{23}$. To assess the potential of this genetic system for CHO cells, the expression levels of NFAT5 under isotonic $(0.3 \mathrm{osm} / \mathrm{kg})$ and hyperosmotic $(0.4 \mathrm{osm} / \mathrm{kg})$ conditions were analyzed. For both $\mathrm{CHO}$ cell derivatives $\mathrm{K} 1$ and DG44, NFAT5 expression was confirmed, even showing a 2.9-fold (CHO-K1) and 2.6-fold (CHO-DG44) elevation of NFAT5 transcripts at hyperosmolality (Fig. 2B).

Since both the presence and functionality of this crucial factor for sensing osmolality were verified, vectors were constructed following the same principle as before by linking a minimal CMV promoter to OREs of AR origin driving the expression of a destabilized BFP variant (FKBP-BFP), including zeocin resistance (Fig. 2C).

After stable transfection of $\mathrm{CHO}-\mathrm{K} 1$ cells with vectors harboring various numbers of OREs, hyperosmotic culture conditions $(0.45 \mathrm{osm} / \mathrm{kg})$ were triggered by $\mathrm{NaCl}$ addition. While $\mathrm{CHO}-\mathrm{K} 1$ mock cells did not induce FKBP-BFP expression and only showed a low basal mean fluorescence of 0.6 (Fig. 2D), introduction of OREs led to a strong fluorescence induction of up to 6.1 mean fluorescence, which was correlated with the ORE number (Fig. 2D). Especially in comparison to isotonic cultured cell lines, an up to 9-fold increased FKBP-BFP signal was obtained for the best construct harboring 7OREs (Fig. 2D). CHO-DG44 cells transfected with either the mock or ORE vectors revealed comparable results with slightly higher basal expression for mock but also showed up to 8.4 mean fluorescence at hyperosmolality for the 7ORE-containing construct (Fig. 2E). In addition, OREs in CHO-DG44 cells led to a 4 - to 5 -fold pronounced FKBP-BFP expression during cultivation in hyperosmotic $(0.45 \mathrm{osm} / \mathrm{kg})$ versus isotonic $(0.32 \mathrm{osm} / \mathrm{kg})$ conditions (Fig. 2E). As osmolality in fermentations is influenced not only by $\mathrm{Na}^{+}$ions of $\mathrm{pH}$ correction agents but also by supplemental feeds, the impact of glucose on the synthetic sensor system was assessed. For both CHO-K1-7ORE and CHO-DG44-7ORE cell lines, a comparable induction with a linear correlation of FKBP-BFP expression to the applied osmolality was detected (Fig. 2F). Finally, the reversibility of FKBP-BFP expression was demonstrated by reversing hyperosmotic conditions to isotonic conditions and nearly restoring the basal expression values of noninduced CHO-K1-7ORE or DG44-7ORE cells. Therefore, the established osmolality sensing system was validated to be suitable for monitoring osmolality during fermentations (Fig. 2G).

Multiplex detection of bioprocess parameters by $\mathrm{CHO}$ sensor cell line. Following the establishment of a synthetic sensor system to monitor oxygen limitation or hyperosmolality by the exploitation of genetic response elements, we aimed to multiplex the inducible reporter systems to sense both parameters simultaneously. Therefore, stable multiplex sensor CHO-K1 and CHO-DG44 cell lines were created expressing both the 5HRE-d2GFP and 7ORE-FKBP-BFP expression vectors (Fig. 3A) and subsequently functionally validated in batch fermentation (Fig. 3A). Growth profiles and viabilities of all batch fermentations are shown in Supplemental Figure 1 . After $48 \mathrm{~h}$, the osmolality was increased from $0.31 \mathrm{osm} / \mathrm{kg}$ to $0.41 \mathrm{osm} / \mathrm{kg}$ to trigger FKBP-BFP expression (Fig. 3B), followed by a reduction in the $\mathrm{pO}_{2}$ set point to $1 \%$ after $72 \mathrm{~h}$ to trigger the HRE-dependent d2GFP signal (Fig. 3C). For both the CHO-K1 and CHO-DG44 sensor cell lines, increased FKBP-BFP expression was measured when osmolality was increased, although CHO-DG44 cells (8.5 mean fluorescence) revealed a stronger FKBP-BFP signal than CHO-K1 cells (3.1 mean fluorescence) (Fig. 3D). In comparison, CHO-K1 and CHO-DG44 control cell lines missing response elements for hypoxia or osmolality maintained low basal expression throughout the whole batch fermentation for both conditions (Fig. 3D,E). Similar to the induction of FKBP-BFP by hyperosmolality, d2GFP expression was also triggered (CHO-K1: 1.9 mean fluorescence; CHODG44: 1.6 mean fluorescence) after establishing oxygen limitations, demonstrating the functionality and the possibility to multiplex response elements to monitor multiple cultivation conditions simultaneously (Fig. 3E). Finally, the reversibility of the system was analyzed by restoring $40 \% \mathrm{pO}_{2}$ after $96 \mathrm{~h}$, which inhibited the expression of $\mathrm{d} 2 \mathrm{GFP}$ and reduced the fluorescence signal for CHO-K1 and -DG44 cell lines to approximately 0.8 and 
A
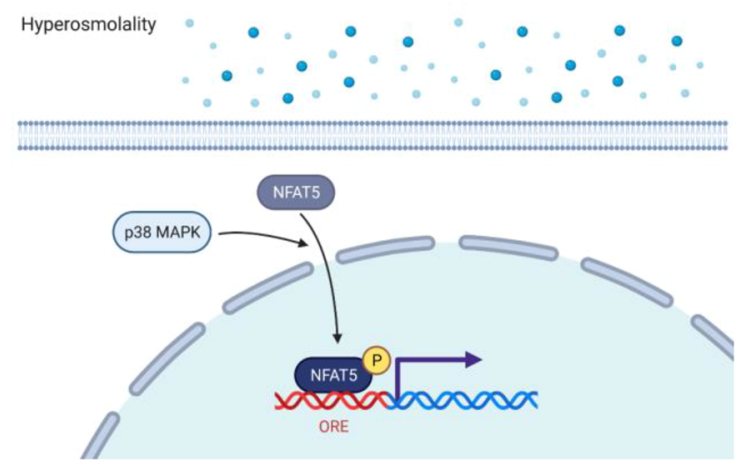

D
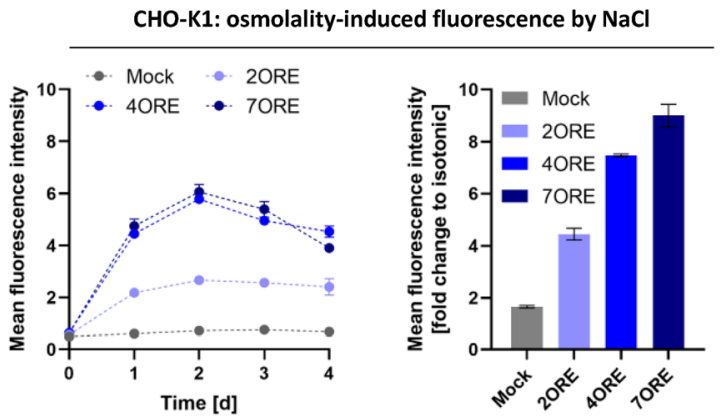

$\mathbf{F}$

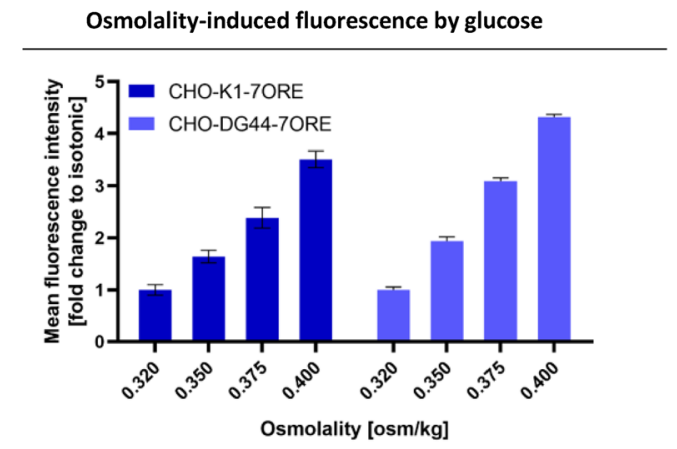

B

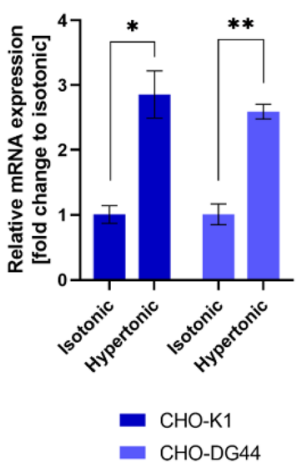

E

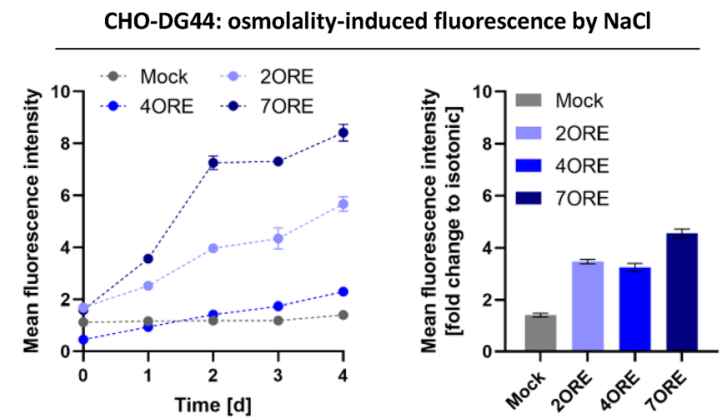

G

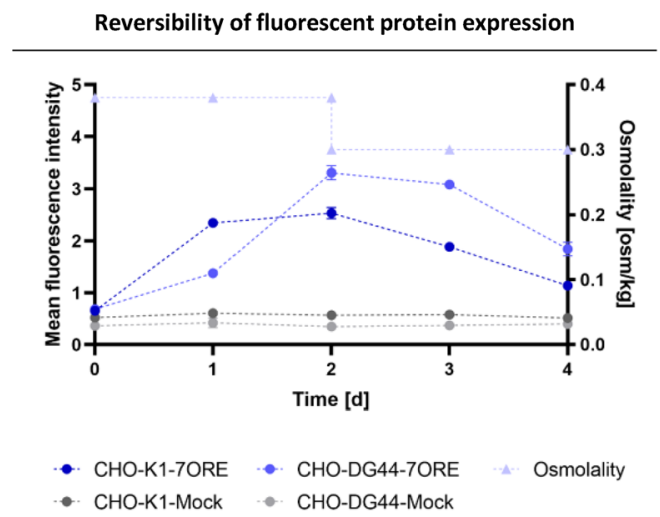

Figure 2. Development of a hyperosmolality-sensitive expression system. (A) Hyperosmolality signaling pathway. (B) qPCR of NFAT5 expression in CHO-K1 and CHO-K1-DG44 cells under isotonic and hyperosmotic conditions. (C) Schematic illustration of the hyperosmolality inducible FKBP-BFP expression vector. (D) Flow cytometric analysis of $\mathrm{NaCl}$-induced hyperosmotic cultured $\mathrm{CHO}-\mathrm{K} 1$ cells (left) and relative fold-change of mean fluorescence against the isotonic cultured control (right). (E) Flow cytometric analysis of $\mathrm{NaCl}$-induced hyperosmotic cultured CHO-DG44 cells (left) and relative fold-change of mean fluorescence against the isotonic cultured control (right). (F) Flow cytometric analysis of glucose-induced hyperosmotic cultured CHO-K1 and -DG44 cells with relative fold-change of mean fluorescence against the isotonic cultured control. (G) Flow cytometric analysis of hyperosmotic cultured CHO-K1 and -DG44 cells with restoration of isotonic conditions after 2 days. [ $\mathrm{n}=3$ replicates, Mean $\pm \mathrm{SD} ;^{*}=p<0.05 ;{ }^{* *}=p<0.01$ ].

1.1 mean fluorescence, respectively (Fig. 3C,E). Overall, CHO-DG44 cells generally display a greater inducibility when comparing the FKBP-BFP or d2GFP signal of 5HRE- and 7ORE-harboring expression cell lines to their equally cultivated controls, which resulted in a significant up to $\sim 6$-fold amplification for both FKBP-BFP and d2GFP after induction (Fig. 3F,G).

Automated real-time monitoring of a $\mathrm{CHO}$ multiplex sensor cell line. After demonstrating the functionality of a multiplex sensor $\mathrm{CHO}$ cell line to monitor hyperosmolality and hypoxia simultaneously during batch fermentation, we created a fully automated in-line system to observe the cellular state close to real- 
A
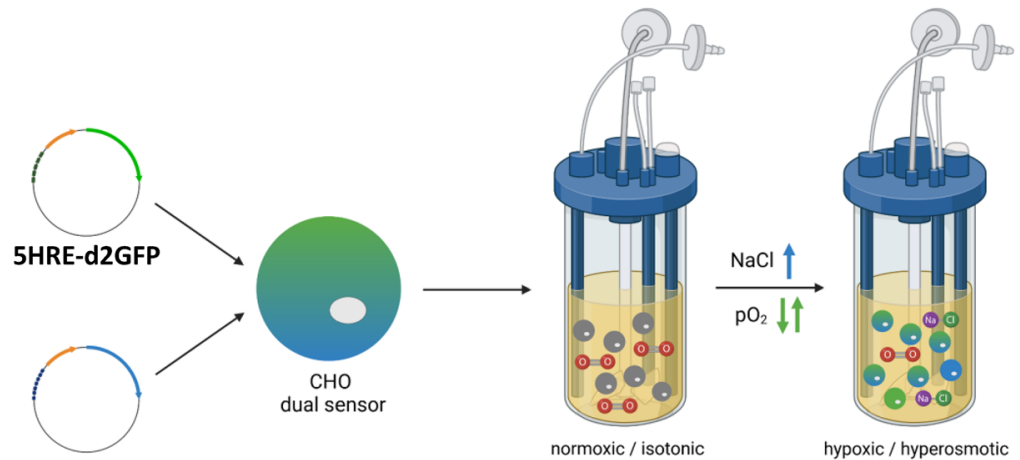

B

Osmolality

C

Partial oxygen pressure
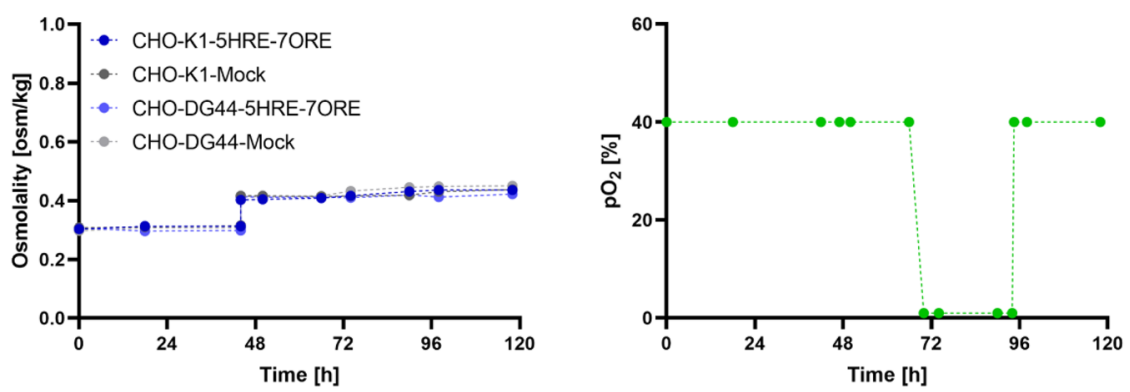

D

BFP fluorescence

E

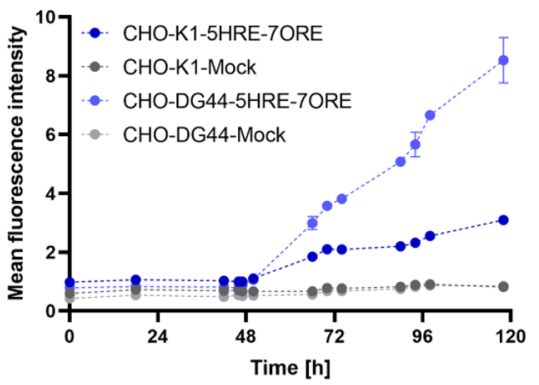

GFP fluorescence

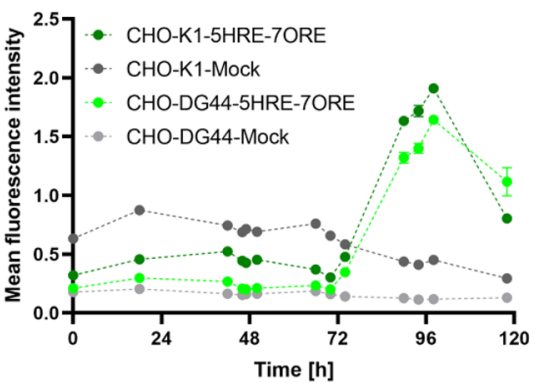

$\mathbf{F}$

G
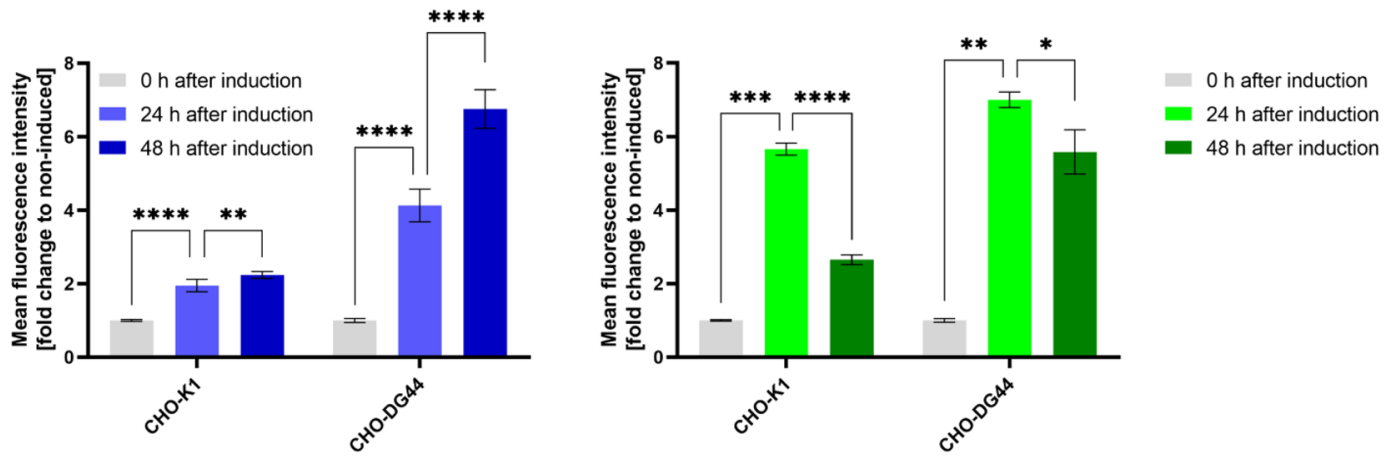

Figure 3. Batch fermentation of dual sensor cell lines under hypoxic and hyperosmotic conditions. (A) Schematic experimental setup. (B) Osmolality during batch fermentation. (C) $\mathrm{pO}_{2}$ concentration during batch fermentation. (D,E) Flow cytometric analysis of CHO-K1 and -DG44 cells during batch fermentation expressing FKBP-BFP (D) and d2GFP (E). (F,G) Relative fold-change of FKBP-BFP (F) or d2GFP (G) mean fluorescence against the mock control cell line during batch fermentation.

time. Therefore, we performed batch fermentation with the above established CHO-DG44 multiplex sensor cell line and used a peristaltic pump controlled by a socket timer to automatically flush the cell culture suspension 
in a $90 \mathrm{~min}$ interval through a flow cell implemented in a fluorescence microscope (Fig. 4A). In a synchronized manner, fluorescence microscopy provided five qualitative images distributed over the flow cell and quantitative cytoimaging results for each time point. For the induction of hypoxia or hyperosmolality, the previously established conditions were applied with a $\mathrm{pO}_{2}$ switch from 40 to $1 \%$ and an increase in osmolality from 0.3 to $0.4 \mathrm{osm} / \mathrm{kg}$ (Fig. 4B).

A decrease in the d2GFP signal was observed to 89 mean fluorescence until the $\mathrm{pO}_{2}$ set point was decreased to $1 \%$ and hypoxia was established (Fig. $4 \mathrm{C}$ ). Subsequently, the d2GFP mean fluorescence increased continuously until $72 \mathrm{~h}$ and restoration of $40 \% \mathrm{pO}_{2}$, where fluorescence started to stagnate at approximately 117 mean fluorescence (Fig. 4C,D). Notably, for d2GFP expression, the measured FKBP-BFP signal also decreased at the beginning of batch fermentation to 115 mean fluorescence until osmolality was adjusted to $0.4 \mathrm{osm} / \mathrm{kg}$ and hyperosmolality was established (Fig. 4C). Thereafter, a comparable nearly linear increase in FKBP-BFP expression was detected, reaching up to 156 mean fluorescence toward the end of the process (Fig. 4C,D). When the oxygen concentration was revered to $40 \% \mathrm{pO}_{2}$ and normoxia was restored, the $\mathrm{d} 2 \mathrm{GFP}$ signal stopped to increase but was not decreasing until a slight decrease of the d2GFP expression was visible during the end of the fermentation. Interestingly, the recorded fluorescence images revealed a heterogenic population of strong- and weak-expressing cells for d2GFP and FKBP-BPF during the different states of induction (Fig. 4D). In this context, green, blue and cyan cells were recorded, indicating cell stress by hypoxia, hyperosmolality or both (Fig. 4D). Finally, fluorescent pictures indicated an attachment of cells in the flow channel between 48 and $72 \mathrm{~h}$, which revealed d2GFP expression due to hypoxic conditions inside chamber and explained the slight decrease of the d2GFP expression after normoxia was restored.

\section{Discussion}

The production of complex biotherapeutics is mainly performed in CHO cells using bioprocesses in large-scale industrial fermenters ${ }^{26}$. To obtain ideal processes, including strong cell growth, high product titer and good product quality, constant monitoring of critical parameters and their maintenance in tight ranges is mandatory ${ }^{1}$.

Our work aimed to establish a novel and innovative solution by generating a self-reporting sensor cell line responding to unfavorable process conditions through the expression of unstable fluorescent proteins. While HREs of VEGF origin in CHO cells were shown to elevate the expression of recombinant proteins during oxygen limitations, HREs can also be utilized to control the expression of destabilized GFP (d2GFP) as a detectable marker for hypoxia. Our results indicate the functionality of HREs in both CHO-K1 and CHO-DG44 cells, with a stronger efficiency of HREs in CHO-DG44 cells. This finding was in concordance with our previous results revealing reduced expression and stabilization of the crucial hypoxia reporter HIF-1 $\alpha$ in CHO-K1 cells in the absence of oxygen ${ }^{4}$. However, inducibility of d2GFP was sufficient for both cell lines to enable monitoring of oxygen limitations by expressing the fluorescent reporter molecule.

To multiplex the detection of several important bioprocess parameters, we also worked on the introduction of OREs to generate hyperosmolality-sensitive $\mathrm{CHO}$ cells.. Although overexpression of NFAT5 under hyperosmolality in $\mathrm{CHO}$ cells was shown before ${ }^{27}$, OREs have not yet been utilized and require detailed testing of several arrangements and conditions to unveil the most potent setup for reporting hyperosmolality by inducible FKBPBFP expression. We were able to identify NFAT5 upregulation during hyperosmolality and achieved a nearly linear correlation of FKBP-BFP expression with both the number of ORE repeats and osmolality. Furthermore, our findings indicate the most promising inducibility by employing 7ORE repeats with comparable results in CHO-K1 and -DG44.

As proof of concept, an automated fluorescence microscopy-based setup was developed, enabling the observation and quantification of both fluorescence proteins in a continuous, close to real-time manner. Since we successfully introduced two independent systems for bioprocess monitoring, the possibility of multiplexing up to four bioprocess conditions with nonoverlapping fluorophores seems feasible when establishing suitable response elements ${ }^{28}$. In particular, the destabilization domain FKBP may be an interesting tool to generate further unstable fluorescent proteins by $\mathrm{N}$-terminal fusion to fluorescent proteins, as applied for BFP ${ }^{29}$. Therefore, available fluorescent proteins such as RFP or miRFP670 could be modified to generate unstable, noninterfering versions for monitoring additional bioprocess conditions, as thus far, no efficient red or near infrared fluorescent proteins are available ${ }^{29-31}$. In this context, it is also important to develop further green or blue fluorescent proteins with even shorter half-life times than the $2 \mathrm{~h}$ of the utilized fluorescent proteins to generate a more precise and functional monitoring system. Although in our experiments restoration of normoxic or isotonic conditions led to decreased fluorescence expression, the basal mean fluorescence signal of noninduced CHO cells was not reached, and faster turnover of the mean fluorescence signal would be necessary for industrial applications. However, over the past years, a broad range of novel fluorescent proteins have been developed for the continuously growing requirements of novel diagnostic or imaging applications ${ }^{32-34}$.

As our study mainly served as a first of its kind proof of concept study, serval points have to be addressed to enable its industrial use like the monitoring speed, reporting strength and precision. Thereby the sensitivity of the system has to be optimized to enable the detection of the reporter molecule as soon as a limitation occurs and as fast as possible before adverse effects affect the cell culture ideally within $2-3 \mathrm{~h}$. Another limitation represents the half-life time of the fluorescent dyes which has to be shorter than $2 \mathrm{~h}$ to reach a higher precision. In addition, the expression of free fluorescent proteins could be problematic for downstream processing (DSP) and may therefore represent an issue for regulatory authorities. Thus, alternative reporting systems are required in the future. Here, fluorophores linked to marker molecules of natural side products of CHO cells as exosomes may represent a potential alternative mediator of unfavorable conditions with less interference in DSP ${ }^{35}$.

As mentioned before, a triple or even quadruple sensor cell line should be feasible when suitable response elements are identified. Glucose deprivation is communicated in nature by the activation of glyconeogenesis, which 
A

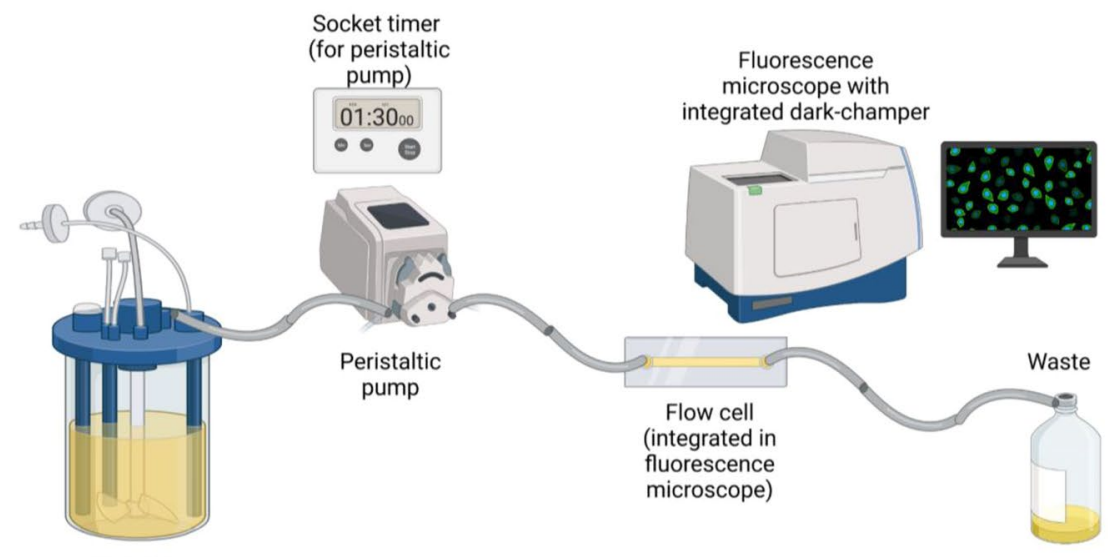

B

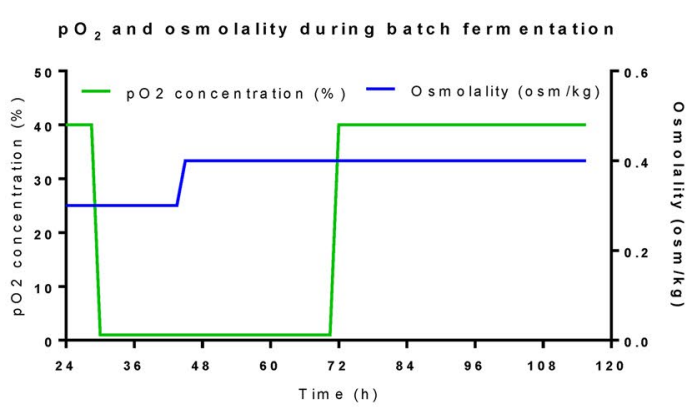

D
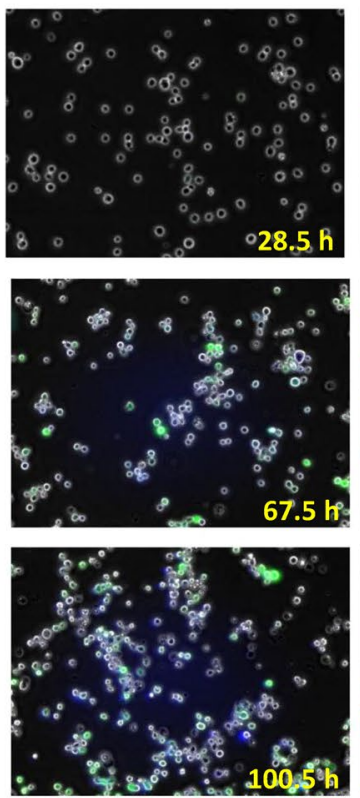
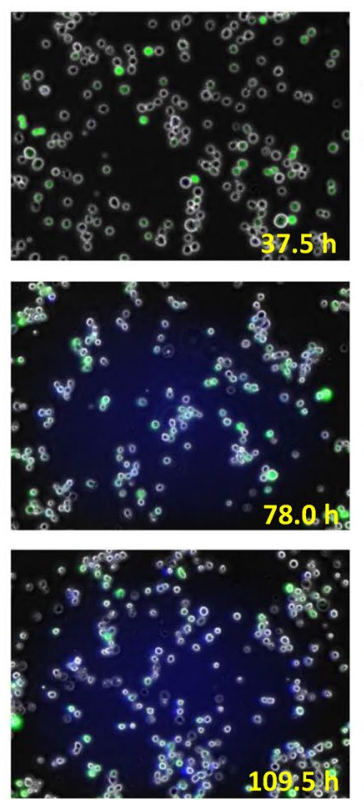

C

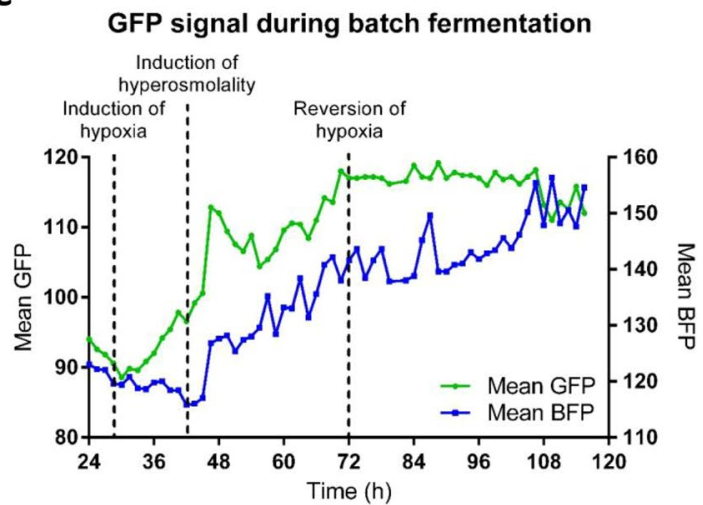

Figure 4. Automated real-time monitoring of a $\mathrm{CHO}$ multiplex sensor cell line during batch fermentation. (A) Schematic experimental setup. (B) Osmolality and $\mathrm{pO}_{2}$ concentration during batch fermentation. (C) d2GFP and FKBP-BFP signals of CHO-DG44 cells during batch fermentation recorded by image cytometry at $90 \mathrm{~min}$ intervals. (D) Representative fluorescence microscopy images of CHO-DG44 cells during batch fermentation recorded at 90 min intervals. 


\begin{tabular}{|l|l|l|l|l|l|}
\hline Name (vector/cell line) & Vector backbone & Fluorophore & Response element & RE repetitions & Selectable marker \\
\hline Mock-GFP & pEF-myc-cyto-mCMV & d2GFP & - & - & Neo \\
\hline 2HRE-GFP & pEF-myc-cyto-mCMV & d2GFP & VEGF-HRE & 2 & Neo \\
\hline 5HRE-GFP & pEF-myc-cyto-mCMV & d2GFP & VEGF-HRE & 5 & Neo \\
\hline 8HRE-GFP & pEF-myc-cyto-mCMV & d2GFP & VEGF-HRE & 8 & Neo \\
\hline Mock-BFP & pEF-myc-cyto-mCMV & FKBP-BFP & - & - & Zeo \\
\hline 2ORE-BFP & pEF-myc-cyto-mCMV & FKBP-BFP & AR-ORE & 2 & Zeo \\
\hline 4 ORE-BFP & pEF-myc-cyto-mCMV & FKBP-BFP & AR-ORE & 4 & Zeo \\
\hline 7ORE-BFP & pEF-myc-cyto-mCMV & FKBP-BFP & AR-ORE & 7 & Zeo \\
\hline
\end{tabular}

Table 1. Generated vector constructs for the analysis of HRE and ORE functionality.

in front of crucial glyconeogenic genes ${ }^{36-39}$. Adaptation of CREs and their linkage to an additional fluorescent protein may enable $\mathrm{CHO}$ cells to report starvation and lead to novel feeding regimes exploiting response elements as tools for automated sensing systems.

\section{Methods}

Cell culture. Suspension-adapted CHO-DG44 (A1100001, Thermo Fisher, Darmstadt, Germany) and CHO-K1 (CCL-61TM, ATCC) cells were cultured in chemically defined HyClone ${ }^{\text {Ta }}$ SFM4CHO ${ }^{\text {Tx }}$ medium (Cytiva, Marlborough, MA, USA) supplemented with $10 \mathrm{~g} / \mathrm{L}$ glucose (Roth, Karlsruhe, Germany) and $4 \mathrm{mM}$ L-glutamine (Lonza, Basel, Switzerland) at $37^{\circ} \mathrm{C}, 5 \% \mathrm{CO}_{2}, 85 \%$ humidity, and $140 \mathrm{rpm}$ (25 mm orbit) in shake flasks (Corning Inc, New York, USA) using a Kuhner incubate shaker (Adolf Kühner AG, Basel, Swiss). Subculturing was performed every 3-4 days to a viable cell density (VCD) of $0.5 \times 10^{6}$ cells $/ \mathrm{mL}$. VCD and viability were determined by trypan blue exclusion using CEDEX XS Cell Analyzer (Roche Diagnostics, Mannheim, Germany). CHO cells were transfected with $15 \mu \mathrm{g}$ vector using the NEON ${ }^{\circledast}$ transfection system (Thermo Fisher Scientific, Waltham, MA, USA). Selection of polyclonal cell lines stably expressing derivatives of the pEF-myccyto-mCMV-d2GFP vector (Addgene, Watertown, MA, USA) was performed using $500 \mu \mathrm{g} / \mathrm{mL}$ G418 sulfate (Genaxxon Bioscience, Ulm, Germany) and/or $400 \mu \mathrm{g} / \mathrm{mL}$ Zeocin (InvivoGen, Toulouse, France). All utilized vectors are listed in Table 1. To induce fluorescent protein expression, $\mathrm{CHO}$ cells were seeded with a VCD of $0.5 \times 10^{6}$ cells $/ \mathrm{mL}$ and grown statically for several days at undefined hypoxic conditions $\left(37^{\circ} \mathrm{C}, 5 \% \mathrm{CO}_{2}\right.$, and $85 \%$ humidity) in a Forma ${ }^{\mathrm{Tm}}$ Steri-Cycle ${ }^{\mathrm{ma}}$ incubator (Thermo Fisher Scientific, Waltham, MA, USA) where a static cultivation and sedimentation of the cells was sufficient to generate hypoxic conditions. In addition, a shaken control batch was inoculated with a VCD of $0.5 \times 10^{6}$ cells $/ \mathrm{mL}$ to meme normoxic conditions. To induce fluorescent protein expression by OREs, hyperosmotic conditions (up to $0.45 \mathrm{osm} / \mathrm{kg}$ ) were created by addition of sodium chloride $(\mathrm{NaCl})$ or glucose and compared to an isotonic $(0.3 \mathrm{osm} / \mathrm{kg})$ cultured control. To analyze the reversibility of the fluorescent protein expression after hyperosmotic induction of $0.4 \mathrm{osm} / \mathrm{kg}$, the cells were centrifuged and the media was changed to isotonic media with $0.3 \mathrm{osm} / \mathrm{kg}$. The osmolality of culture supernatants was measured using OSMOMAT 030 (Gonotec, Berlin, Germany).

Batch fermentation. For cultivation at defined $\mathrm{O}_{2}$ concentrations and hyperosmotic conditions, $\mathrm{CHO}$ cells were inoculated at a VCD of $0.7 \times 10^{6}$ cells $/ \mathrm{mL}$ in a volume of $1 \mathrm{~L}$ using $2 \mathrm{~L}$ stirred tank benchtop bioreactors (Sartorius, Göttingen, Germany). Process conditions were controlled at a stirring speed of $100 \mathrm{rpm}, \mathrm{pH}$ of 7.15 , and temperature of $37^{\circ} \mathrm{C}$. The $\mathrm{O}_{2}$ set point was adjusted at different time points by gassing with $\mathrm{N}_{2}$ to reach a $\mathrm{pO}_{2}$ of $1 \%$. To restore normoxia $\left(\mathrm{pO}_{2}=40 \%\right)$ the gas mix was adjusted by the fermentation head-unit and air was used to enhance the desired $\mathrm{pO}_{2}$. If air was not sufficient to provide the desired $\mathrm{O}_{2}$ concentration, the fermentation head-unit mixed pure $\mathrm{O}_{2}$ into the gas mix. Osmolality was increased by the addition of a $3 \mathrm{M} \mathrm{NaCl}$ solution with a peristaltic pump and the volume of added $\mathrm{NaCl}$ was observed by a scale. We aimed to provide an increased osmolality of around $0.4 \mathrm{osm} / \mathrm{kg}$ and confirmed a final osmolality after $\mathrm{NaCl}$ addition of $0.41 \mathrm{osm} / \mathrm{kg}$ by external measurement of the cell culture suspension.

Molecular biology. The starting plasmid 5HRE/GFP was a gift from Martin Brown \& Thomas Foster (Addgene plasmid \# 46926) ${ }^{40}$. Constructs were cloned using HRE sequences of VEGF and ORE sequences of AR published by Javan et al. $2017^{16}$ and Ferraris et al. $1999^{41}$, respectively. The oligos used are listed in Table 2. Prior to cloning, oligonucleotides were annealed by heating to $95^{\circ} \mathrm{C}$ followed by slow cooling to $25^{\circ} \mathrm{C}$, phosphorylation using T4 PNK (New England Biolabs, Ipswich, MA, USA), and ligation by T4 ligase (New England Biolabs, Ipswich, MA, USA). After removing the 5HREs by digestion with XhoI and BglII (both New England Biolabs, Ipswich, MA, USA), the randomly ligated double-stranded oligonucleotides were cloned into the pEF-myccyto-mCMV-d2GFP vector. The origin of the destabilized blue fluorescent protein variant FKBP-mTagBFP2 and zeocin resistance for the exchange of fluorescent protein and selectable markers were the vectors pAW63.YY1. FKBP.knock-in. BFP (Addgene plasmid \#104371) ${ }^{29}$ and pcDNA3.1/Zeo(+) (Thermo Fisher Scientific, Waltham, MA, USA), respectively. FKBP-mTagBFP2 and zeocin resistance were amplified via PCR using the following primers: FKBP-BFP Fwd (5-ATATCCATGGGTGCCCCTTCGACGGTTGTA-3); FKBP-BFP Rev (5-ATA TTCTAGACTTCCCGGGTCGAGAAGGTC-3); Zeo Fwd (5'-ATATTCTAGACCCGTTTAAACCCGCTGA3'); and Zeo Rev (5'-ATATTTCGAACTTTCATAGAAGGCGGCGGT-3'). Cloning of fluorescent protein and 


\begin{tabular}{|l|l|l|}
\hline Oligonucleotide & Sequence $\left[\mathbf{5}^{\prime} \rightarrow \mathbf{3}^{\prime}\right]$ & Gene \\
\hline Mock Fw & TCGAGACTAGTCCAGTGA & - \\
\hline Mock Rev & GATCTCACTGGACTAGTC & - \\
\hline VEGF-HRE-pair 1 Fw & TCGAGCCACAGTGCATACGTGGGCTCCAACAGGTCCTCTT & VEGF \\
\hline VEGF-HRE-pair 1 Rev & CTCGACAAGAGGACCTGTTGGAGCCCACGTATGCACTGTGGC & VEGF \\
\hline VEGF-HRE-pair 2 Fw & GTCGAGCCACAGTGCATACGTGGGCTCCAACAGGTCCTCTT & VEGF \\
\hline VEGF-HRE-pair 2 Rev & CTCGACAAGAGGACCTGTTGGAGCCCACGTATGCACTGTGG & VEGF \\
\hline VEGF-HRE-pair 3 Fw & GTCGAGCCACAGTGCATACGTGGGCTCCAACAGGTCCTCTTGTCGA & VEGF \\
\hline VEGF-HRE-pair 3 Rev & GATCTCGACAAGAGGACCTGTTGGAGCCCACGTATGCACTGTGG & VEGF \\
\hline AR-ORE-pair 1 Fw & TCGAGTGGAAAATCACC & AR \\
\hline AR-ORE-pair 1 Rev & CTCGACGGTGATTTTCCAC & AR \\
\hline AR-ORE-pair 2 Fw & GTCGAGTGGAAAATCACC & AR \\
\hline AR-ORE-pair 2 Rev & CTCGACGGTGATTTTCCA & AR \\
\hline AR-ORE-pair 3 Fw & GTCGAGTGGAAAATCACCGTCGA & AR \\
\hline AR-ORE-pair $3 \mathrm{Rev}$ & GATCTCGACGGTGATTTTCCA & AR \\
\hline
\end{tabular}

Table 2. Oligonucleotide sequences for molecular biology.

selectable markers into the pEF-myc-cyto-mCMV backbone was performed after digestion with NcoI and XbaI as well as $\mathrm{XbaI}$ and BstBI (New England Biolabs, Ipswich, MA, USA).

RNA isolation. Total RNA was isolated from $3 \times 10^{6}$ cells using the miRNeasy Kit (Qiagen, Hilden, Germany) according to the manufacturer's protocol. RNA concentration and purity were determined using a NanoDrop $^{\text {re }} 1000$ Spectrophotometer (Thermo Fisher Scientific, Waltham, MA, USA) by absorbance at $260 \mathrm{~nm}$.

RT-PCR. After RNA isolation, reverse transcription to cDNA was performed with the High Capacity cDNA Reverse Transcription Kit (Thermo Fisher Scientific, Waltham, MA, USA) according to the manufacturer's protocol. Quantitative real-time PCR on NFAT5 and GAPDH as a loading control was performed using GreenMasterMix No ROX (Genaxxon Bioscience, Ulm, Germany) and the following primers: NFAT5 Fw (5'-TACCAC GGACAACAAAGGCA-3'); NFAT5 Rev (5'-AAGTCGATGCCCTTCAGCTC-3'); GAPDH Fw (5'- GACTCT ACCCATGGCAAGTTCA-3'); and GAPDH Rev (5'-TCGCTCCTGGAAGATGGTGATG-3'). A LightCycler ${ }^{\circledR}$ 480 Instrument II (Roche Diganostics, Mannheim, Germany) was used for gene expression analysis.

Flow cytometry analysis. The mean fluorescence intensity of d2EGFP and FKBP-mTagBFP2 expressed under hypoxic and hyperosmotic conditions, respectively, was measured using MACSQuant Analyzer 10 (Miltenyi Biotec, Bergisch-Gladbach, Germany). D2EGFP was measured using a $488 \mathrm{~nm}$ laser and the 525/50 nm filter setting (Channel B1), and FKBP-mTagBFP2 was detected by a $405 \mathrm{~nm}$ laser and a 450/50 nm filter setting (Channel V1). Subsequent data analysis was performed with MACSQuantify ${ }^{\text {Tix }}$ Software.

Live fluorescence microscopy. For live fluorescence microscopy, CHO cells were cultivated in a 2 L bioreactor, and deprivation conditions were induced as described in the methods section Batch fermentation. Automated and continuous sampling was performed by utilizing a peristaltic pump controlled by a socket timer to sample the cell culture suspension every $1 \mathrm{~h} 30 \mathrm{~min}$. Fluorescence microscopy was performed by integrating a coated $\mu$-slide with Luer adapters and a $0.2 \mathrm{~mm}$ channel height (Ibidi GmbH, Gräfelfing, Germany) into a BZ X 800 fluorescence microscope (Keyence, Neu-Isenburg, Germany). Images were automatically recorded by fluorescence microscopy at 5 fixed points in the $\mu$-slide every $1 \mathrm{~h} 30 \mathrm{~min}, 3 \mathrm{~min}$ after the peristaltic pump stopped sampling to ensure clear good-quality pictures. Fluorescence intensity was qualitatively analyzed by imaging cytometry using Keyence BZ X 800 Analyzing software.

Statistical analysis. Two-tailed unpaired t test with Welsh's correction was applied for statistical analysis using GraphPad Prism 6. Data are presented as the mean \pm standard deviation.

Received: 24 October 2021; Accepted: 25 January 2022

Published online: 10 February 2022

\section{References}

1. Rathore, A. S., Mishra, S., Nikita, S. \& Priyanka, P. Bioprocess control: Current progress and future perspectives. Life 11, 557 (2021).

2. Trummer, E. et al. Process parameter shifting: Part I. Effect of DOT, pH, and temperature on the performance of Epo-Fc expressing CHO cells cultivated in controlled batch bioreactors. Biotechnol. Bioeng. 94, 1033-1044 (2006).

3. Li, F., Vijayasankaran, N., Shen, A., Kiss, R. \& Amanullah, A. Cell culture processes for monoclonal antibody production. MAbs 2, 466-479 (2010). 
4. Zeh, N. et al. Cell line development for continuous high cell density biomanufacturing: Exploiting hypoxia for improved productivity. Metab. Eng. Commun. 13, e00181 (2021).

5. Chance, B. Reaction of oxygen with the respiratory chain in cells and tissues. J. Gen. Physiol. 49(Suppl), 163-195 (1965).

6. Hubbi, M. E. \& Semenza, G. L. Regulation of cell proliferation by hypoxia-inducible factors. Am. J. Physiol. Physiol. 309, C775-C782 (2015).

7. Hubbi, M. E. \& Semenza, G. L. An essential role for chaperone-mediated autophagy in cell cycle progression. Autophagy 11, 850-851 (2015).

8. Paul, A. J., Handrick, R., Ebert, S. \& Hesse, F. Identification of process conditions influencing protein aggregation in Chinese hamster ovary cell culture. Biotechnol. Bioeng. 115, 1173-1185 (2018).

9. Bickel, F. et al. Reversible NaCl-induced aggregation of a monoclonal antibody at low $\mathrm{pH}$ : Characterization of aggregates and factors affecting aggregation. Eur. J. Pharm. Biopharm. 107, 310-320 (2016).

10. Zhu, M. M. et al. Effects of elevated pCO2 and osmolality on growth of $\mathrm{CHO}$ cells and production of antibody-fusion protein $\mathrm{B} 1$ : A case study. Biotechnol. Prog. 21, 70-77 (2008).

11. Alhuthali, S., Kotidis, P. \& Kontoravdi, C. Osmolality effects on $\mathrm{CHO}$ cell growth, cell volume, antibody productivity and glycosylation. Int. J. Mol. Sci. 22, 3290 (2021).

12. Li, F. et al. A systematic approach for scale-down model development and characterization of commercial cell culture processes. Biotechnol. Prog. 22, 696-703 (2006).

13. Tang, P. et al. Kinetic modeling of Chinese hamster ovary cell culture: factors and principles. Crit. Rev. Biotechnol. 40, 265-281 (2020).

14. Lee, P., Chandel, N. S. \& Simon, M. C. Cellular adaptation to hypoxia through hypoxia inducible factors and beyond. Nat. Rev. Mol. Cell Biol. 21, 268-283 (2020).

15. Gonzalez, F. J., Xie, C. \& Jiang, C. The role of hypoxia-inducible factors in metabolic diseases. Nat. Rev. Endocrinol. 15, 21-32 (2019).

16. Javan, B. \& Shahbazi, M. Hypoxia-inducible tumour-specific promoters as a dual-targeting transcriptional regulation system for cancer gene therapy. Ecancermedicalscience 11, 751 (2017).

17. Stockmann, C. \& Fandrey, J. Hypoxia-induced erythropoietin production: A paradigm for oxygen-regulated gene expression. Clin. Exp. Pharmacol. Physiol. 33, 968-979 (2006).

18. Kimura, H. et al. Identification of hypoxia-inducible factor 1 ancillary sequence and its function in vascular endothelial growth factor gene induction by hypoxia and nitric oxide. J. Biol. Chem. 276, 2292-2298 (2001).

19. Bergeron, M. et al. Role of hypoxia-inducible factor-1 in hypoxia-induced ischemic tolerance in neonatal rat brain. Ann. Neurol. 48, 285-296 (2000).

20. Lee, J. W., Ko, J., Ju, C. \& Eltzschig, H. K. Hypoxia signaling in human diseases and therapeutic targets. Exp. Mol. Med. 51, 1-13 (2019).

21. Miyakawa, H., Woo, S. K., Dahl, S. C., Handler, J. S. \& Kwon, H. M. Tonicity-responsive enhancer binding protein, a Rel-like protein that stimulates transcription in response to hypertonicity. Proc. Natl. Acad. Sci. 96, 2538-2542 (1999).

22. Yang, B. et al. Elevated activity of transcription factor nuclear factor of activated T-cells 5 (NFAT5) and diabetic nephropathy. Diabetes 55, 1450-1455 (2006)

23. Aramburu, J. et al. Regulation of the hypertonic stress response and other cellular functions by the Rel-like transcription factor NFAT5. Biochem. Pharmacol. 72, 1597-1604 (2006).

24. Lee, J. H. et al. NFAT5 induction and its role in hyperosmolar stressed human limbal epithelial cells. Investig. Ophthalmol. Vis. Sci. 49, 1827-1835 (2008).

25. Chua, O. W. H. et al. Role of nuclear factor of activated T-cells 5 in regulating hypertonic-mediated secretin receptor expression in kidney collecting duct cells. Biochim. Biophys. Acta Gene Regul. Mech. 1859, 922-932 (2016).

26. Wurm, F. M. Production of recombinant protein therapeutics in cultivated mammalian cells. Nat. Biotechnol. 22, 1393-1398 (2004).

27. Pfizenmaier, J., Junghans, L., Teleki, A. \& Takors, R. Hyperosmotic stimulus study discloses benefits in ATP supply and reveals miRNA/mRNA targets to improve recombinant protein production of CHO cells. Biotechnol. J. 11, 1037-1047 (2016).

28. Mathias, S. et al. Visualisation of intracellular production bottlenecks in suspension-adapted CHO cells producing complex biopharmaceuticals using fluorescence microscopy. J. Biotechnol. 271, 47-55 (2018).

29. Weintraub, A. S. et al. YY1 is a structural regulator of enhancer-promoter loops. Cell 171, 1573-1588.e28 (2017).

30. Chu, B. W., Banaszynski, L. A., Chen, L. \& Wandless, T. J. Recent progress with FKBP-derived destabilizing domains. Bioorg. Med. Chem. Lett. 18, 5941-5944 (2008).

31. Iwamoto, M., Björklund, T., Lundberg, C., Kirik, D. \& Wandless, T. J. A general chemical method to regulate protein stability in the mammalian central nervous system. Chem. Biol. 17, 981-988 (2010).

32. Hötzer, B., Medintz, I. L. \& Hildebrandt, N. Fluorescence in nanobiotechnology: Sophisticated fluorophores for novel applications. Small 8, 2297-2326 (2012).

33. Liu, P., Mu, X., Zhang, X.-D. \& Ming, D. The near-infrared-II fluorophores and advanced microscopy technologies development and application in bioimaging. Bioconjug. Chem. 31, 260-275 (2020).

34. Drakopoulos, A. \& Decker, M. Development and biological applications of fluorescent opioid ligands. ChemPlusChem 85, 1354$1364(2020)$

35. Keysberg, C. et al. Exploring the molecular content of $\mathrm{CHO}$ exosomes during bioprocessing. Appl. Microbiol. Biotechnol. 105, 3673-3689 (2021).

36. Thonpho, A., Sereeruk, C., Rojvirat, P. \& Jitrapakdee, S. Identification of the cyclic AMP responsive element (CRE) that mediates transcriptional regulation of the pyruvate carboxylase gene in HepG2 cells. Biochem. Biophys. Res. Commun. 393, 714-719 (2010).

37. Stark, R. et al. A role for mitochondrial phosphoenolpyruvate carboxykinase (PEPCK-M) in the regulation of hepatic gluconeogenesis. J. Biol. Chem. 289, 7257-7263 (2014).

38. Jitrapakdee, S. \& Wallace, J. C. Structure, function and regulation of pyruvate carboxylase. Biochem. J. 340(Pt 1), 1-16 (1999).

39. Thiel, G., Al Sarraj, J. \& Stefano, L. cAMP response element binding protein (CREB) activates transcription via two distinct genetic elements of the human glucose-6-phosphatase gene. BMC Mol. Biol. 6, 2 (2005).

40. Vordermark, D., Shibata, T. \& Brown, J. M. Green fluorescent protein is a suitable reporter of tumor hypoxia despite an oxygen requirement for chromophore formation. Neoplasia 3, 527-534 (2001).

41. Ferraris, J. D., Williams, C. K., Ohtaka, A. \& García-Pérez, A. Functional consensus for mammalian osmotic response elements. Am. J. Physiol. Physiol. 276, C667-C673 (1999).

\section{Acknowledgements}

We would like to thank Keyence and especially Noah Kurz for providing the fluorescence microscope BZ-X800 and enabling the automated observation of the fluorescent $\mathrm{CHO}$ sensor cell line. Schematic figures were created using BioRender software. 


\section{Author contributions}

The shared first authors N.Z. and M.B. contributed equally to the study. Both authors are allowed to put their names first in their curriculum vitae. N.Z.: Conceptualization, Methodology, Investigation, Supervision, Writing - Original draft; M.B.: Conceptualization, Methodology, Investigation, Writing - Original draft; N.R.: Investigation, Writing - Review \& Editing; R.H.: Investigation, Writing - Review \& Editing; K.O.: Conceptualization, Supervision, Writing - Review \& Editing.

\section{Funding}

Open Access funding enabled and organized by Projekt DEAL. Nikolas Zeh is funded by the German Federal Ministry of Education and Research, funding program Forschung an Fachhochschulen, contract numbers 13FH162PA6.

\section{Competing interests}

The authors declare no competing interests.

\section{Additional information}

Supplementary Information The online version contains supplementary material available at https://doi.org/ 10.1038/s41598-022-06272-x.

Correspondence and requests for materials should be addressed to N.Z.

Reprints and permissions information is available at www.nature.com/reprints.

Publisher's note Springer Nature remains neutral with regard to jurisdictional claims in published maps and institutional affiliations.

(c) (i) Open Access This article is licensed under a Creative Commons Attribution 4.0 International License, which permits use, sharing, adaptation, distribution and reproduction in any medium or format, as long as you give appropriate credit to the original author(s) and the source, provide a link to the Creative Commons licence, and indicate if changes were made. The images or other third party material in this article are included in the article's Creative Commons licence, unless indicated otherwise in a credit line to the material. If material is not included in the article's Creative Commons licence and your intended use is not permitted by statutory regulation or exceeds the permitted use, you will need to obtain permission directly from the copyright holder. To view a copy of this licence, visit http://creativecommons.org/licenses/by/4.0/.

(c) The Author(s) 2022 